${ }^{6}$ Näätänen, R, and Summala, H, Road-user Behaviour and Traffic Accidents. Amsterdam, North-Holland Publishing Company, 1976.

7 Sabey, B E, and Staughton, G C, paper presented to fifth international conference of the International Association for Accident and Traffic Medicine, London, September 1975.

${ }^{8}$ Gissane, W, Bull, J P, and Roberts, B, Injury, 1970, 1, 195.

${ }^{9}$ Bull, J P, Accident Analysis and Prevention, 1975, 7, 249.

10 Tonge, J I, et al, Medicine, Science and the Law, 1977, 17, 9.
11 Baker, S P, et al, fournal of Trauma, 1974, 14, 187

12 Gögler, E, Series Chirurgica Geigy, No 5. Basle, J A Geigy SA, 1962 (English translation 1965).

13 Galasko, C S B, and Edwards, D H, Injury, 1974, 6, 107.

${ }_{14}$ Smeed, R J, Zeitschrift für Verkehrssicherheit, 1974, 20, 95.

15 Jacobs, G D, and Sayer, I A, A Study of Road Accidents in Selected Urban Areas in Developing Countries, Laboratory Report 775. Crowthorne, TRRL, 1977.

\title{
Epidemiology for the Uninitiated
}

\section{Conduct of surveys}

\author{
GEOFFREY ROSE, D J P BARKER
}

British Medical fournal, 1978, 2, 1201-1202

Anyone who has attempted a study using ordinary clinical case notes knows the problems of unstandardised recording and missing information. For most epidemiological studies it is essential to have purpose-designed records.

\section{Record design}

The aims of the design are to help standardisation, speed, and accuracy in recording under field conditions, and coding and retrieval of results afterwards. Writing takes time, and, where possible, non-numerical information should be ringed or ticked rather than written out. The layout should facilitate subsequent numerical coding and data extraction, with one answer box for each item of information. Copying takes time and may introduce errors; if the record can be precoded, results may go straight to the analysis. An orderly and uncluttered layout makes for fewer mistakes, in both the field and the analysis: results should be vertically aligned on the right of the page, well separate from questions and instructions.

The record starts with the subject's serial number in the study, followed by sufficient personal identification to permit any planned follow-up (address for postal contact, full name, date of birth, and-if available-NHS number for later tracing of morbidity through general practitioners, or mortality through the NHS Central Registry). If general practitioner or hospital follow-up is envisaged, the subject's consent should be recorded on the initial record.

Records should be pretested, both in the field on representative subjects and in the office for subsequent coding and data extraction. It is impossible to foresee all the practical snags. In large studies the record design should be discussed with the statistician who will later be concerned in the analysis.

Department of Medical Statistics and Epidemiology, London School of Hygiene and Tropical Medicine, London WC1E 7HT

GEOFFREY ROSE, DM, FRCP, professor of epidemiology

Faculty of Medicine, University of Southampton, Southampton General Hospital, Southampton S09 4XY

D J P BARKER, PHD, MRCP, reader in clinical epidemiology

\section{QUESTIONNAIRES}

The contrasts of clinical and epidemiological methods are epitomised by the approach to history taking. Clinically it calls for the highest skill-in establishing rapport with the patient, choice of questions, and distinguishing vague from convincing answers. In epidemiology, unfortunately, all unstandardisable skills must be excluded if comparisons are to be valid. Nevertheless, a good questionnaire is not so inferior to a clinical interview as might be imagined, for it concentrates on those few items which are most discriminating, and eliminates what is superfluous; and for these key items the questions are phrased with maximum conciseness and clarity.

Closed-ended questions, with one box for each possible answer (including "don't know"), are more readily answered and classified. Two short questions, each covering one point, are better than one longer question which covers two points at once. Questions which seem clear to a doctor may be difficult or understood differently by the subject, and pretesting is essential. Interviewers must keep strictly to the questions as printed and avoid supplementary questions if possible. Observer variation may be avoided altogether by using self-administered questionnaires.

\section{Staff and training}

In a small study the doctor himself may do all the work, but in large surveys he will need helpers. If an epidemiological examination technique requires skill and clinical judgment, it has probably been insufficiently standardised: if it is adequately standardised, it can usually be taught to any intelligent person.

The figure shows how two observers had distinct but opposite time trends in their performances during the early stages of a survey of skinfold thickness. Such training effects, which are common, should have been completed before the start of the main study: new staff need supervised practice under realistic field conditions.

Despite all precautions, observer differences may persist. Observers should therefore be allocated to subjects in a more or less random way: if one person examined, for example, most of the men, and another most of the women, then observer differences would be confounded with true sex differences. To maintain quality control throughout the survey each examiner's identity should be entered on the record-results for different examiners may then be compared. 


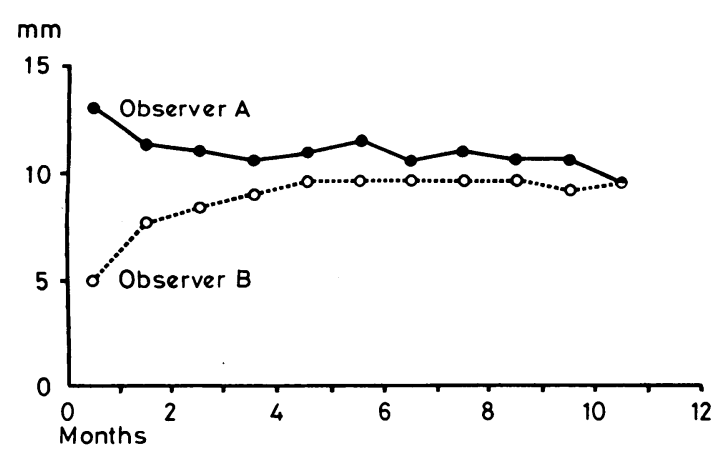

Trend in mean values for triceps skinfold thickness obtained by two observers in the same survey.

\section{Recruitment of subjects}

At the MRC Epidemiology Unit in South Wales (a pattern setter in conducting surveys) a response rate below $95 \%$ is regarded as poor. Most people are willing to take part in medical surveys provided that they trust the investigator, just as patients will nearly always help their own doctor in his research. In population studies, however, there has usually been no previous contact. The selected subjects need an explanation of the purpose of the study, why they in particular have been asked to take part, what is expected from them, and what if anything they will get out of it (a medical check-up, or a report on the research findings). Local general practitioners, too, need to know what is going on. Time given to public relations preparations is always well spent.

Response must be made as easy as possible. If attendance at a centre is required, it is better to send everyone an appointment than to expect them to reply to a letter asking whether they are willing to attend. Transport may sometimes be needed. Often the difference between a mediocre response and a good one is tactful persistence, including second invitations (by recorded delivery), telephone calls, identifying the reasons for non-attendance, and home visits.

\section{RESPONSE RATES}

What response rate is acceptable? For an uncommon condition a response rate of $85 \%$ might be unacceptable, because a handful of cases in the unexamined $15 \%$ might greatly alter the findings; on the other hand, in a survey of smoking habits this response might be considered good.

In addition to prevalence, the acceptable level of response depends on the amount of bias. Non-respondents, and late respondents, tend to differ from those who came at first invitation. Those who did not reply to the first British doctors smoking study subsequently had a mortality rate $40 \%$ higher than those who returned their questionnaires. It is helpful to have some measure of the amount of such bias. Two approaches are possible. Firstly, a small random sample is drawn from the non-respondents, and particularly vigorous efforts are then made to encourage their participation, including home visits; the findings will indicate the extent of bias among non-respondents as a whole. Secondly, some information must be available for all persons listed in the study population; from this it will be possible to contrast respondents and non-respondents with respect to basic characteristics such as age, sex, and residence.

\section{Analysing results}

Epidemiological surveys quickly amass a lot of data. A study of 500 persons could easily yield 50000 items of information, and for large studies there would nowadays be no alternative to computer analysis. To begin with, the coded data are transferred on to 80-column punch cards, where each column records one item of information whose value can range from 0 to 9 . If the results of one subject occupy more than one card, then each card must have space for the subject's identification and for the card number $(1,2$, and so on).

Like gramophone records, punch cards are a curious survival from an earlier electromechanical era. They are read only once into the computer, whose first task is usually to transfer the information to magnetic tape (or disc); if the data are to be rerun on another occasion, then the tape and not the cards will provide the input. Library programs are widely available for the input and analysis of survey results, and in academic centres there will be no problems; for service computer units, however, there may be difficulties in obtaining and manipulating unfamiliar programs. The first essential always is for the user to know exactly what he needs. Some familiarity with survey analysis will help him to request tabulations which are simple to obtain rather than those which demand much special programming.

Many small surveys can be adequately analysed by manual tabulation of results and a desk calculator. The investigator gets the real "feel" of the data, which in a computer are instantly lost to sight. Moreover, programmable calculators take much of the tedium out of statistical calculations. Unfortunately, the data have to be read in afresh for each calculation, and if multiple analyses are needed a computer is still the method of choice.

Eventually this series will be collected into a book and hence no reprints will be available from the authors.

How dangerous is it to eat vegetables grown in a garden plot previously the site of a bonfire on which numerous lead pipes were inadvertently burnt?

If the burning of lead pipes on the bonfire occurred only once the metallic lead would be easily removed from the site and would not be absorbed in any appreciable amount by the soil or plants grown in it. If, however, the burning of lead pipes took place frequently on the site then the concentration of oxides left in the soil could be relatively high, and the soil and any vegetables grown on the garden plot should be analysed for lead. Lead is not normally well absorbed by plants, and most of the lead that is absorbed is retained in the roots. Contamination in root vegetables is mostly on the surface, and any risk is removed by peeling the vegetables. Lettuce and cabbage and other brassicas seem to absorb the highest percentage of lead from the soil and would be the most likely vegetables to cause any risk to health. Adults normally absorb only about $10 \%$ of any ingested lead in food, but children, particularly in infancy, may absorb as much as half of the lead content of the food.

While lead levels in food should be within the limits recommended by the Department of the Environment, these have a fairly large safety margin and are based on all foods containing these amounts of lead. To eat a small quantity of home-grown vegetables, even though these should contain up to 10 times the recommended levels of lead, is most unlikely to cause any harm. Work carried out by Barltrop ${ }^{1}$ in Derbyshire has not shown significant increases in the blood lead of children, even when the natural background levels of lead in the soil reach as high as $1 \%$.

${ }^{1}$ Barltrop, D, et al, Environmental Health Perspective, May 1974, p 75.

An 18-year-old woman has the Wolff-Parkinson-White syndrome but is otherwise fit. Is there any contraindication to oral contraception? Her only medication is a beta-blocker, which she takes three times a day.

I know of no contraindication to using oral contraceptives in such a patient, and I would not expect any interaction to occur with her beta-blocker. 